\title{
Effect of A Polyphenol-Rich Canarium album Extract on the Composition of the Gut Microbiota of Mice Fed a High-Fat Diet
}

\author{
Ning-Ning Zhang ${ }^{(\mathbb{D}}$, Wen-Hui Guo, Han Hu, A-Rong Zhou, Qing-Pei Liu, Bao-Dong Zheng * (D) \\ and Shao-Xiao Zeng * \\ College of Food Science, Fujian Agriculture and Forestry University, Fuzhou 350002, China; \\ znnfst@163.com (N.-N.Z.); gwhfst@163.com (W.-H.G.); hhh3661@163.com (H.H.); zar0306@163.com (A.-R.Z.); \\ jasonlau2013@163.com (Q.-P.L.) \\ * Correspondence: zbdfst@163.com (B.-D.Z.); zsxfst@163.com (S.-X.Z.); Tel.: +86-137-050-09016 (B.-D.Z.); \\ +86-135-093-74665 (S.-X.Z.)
}

Received: 12 August 2018; Accepted: 28 August 2018; Published: 30 August 2018

\begin{abstract}
This study investigated the influence of Canarium album extract (CAext) on intestinal microbiota composition of mice fed a high-fat diet (HFD). Kun Ming (KM) mice were fed either a normal chow diet or a HFD for six weeks. At the seventh week, HFD-fed mice were gavaged daily with saline, or a different dose of CAext for four weeks, respectively. Then, the composition of the gut microbiota was analyzed by high-throughput sequencing technology. Analysis of fecal microbial populations, grouped by phyla, showed significant increases of Firmicutes and Verrucomicrobia, but a decrease of Bacteroidetes in all CAext-fed mice. Particularly, CAext gavage in a low dose or a medium dose caused a significant increase in the proportion of Akkermansia. These findings suggested that CAext can alter the gut microbiota composition of HFD-fed mice, and had a potential prebiotic effects on Akkermansia.
\end{abstract}

Keywords: Canarium album; polyphenol; gut microbiota; high-fat diet

\section{Introduction}

The mammalian gut is inhabited by a vast number of microorganisms [1]. It has been well recognized that a balanced gut microbiota composition confers benefits to the host, whereas gut microbiota imbalance may lead to various metabolic diseases [2]. As the composition of diet has significant effects on gut microbiota, the interest in food-based strategies able to modulate the gut microbiota composition, and probably their functional effects, has rapidly increased in recent years [3]. It has been reported that plant-derived polysaccharide foods increase colonic bifidobacterial numbers [4], whereas a long-term diet rich in saturated fat, such as high-fat diet (HFD), increases the proportion of Firmicutes to Bacteroidetes, which is associated with obesity-induced metabolic diseases [5-7].

Polyphenols are poorly absorbed by the small intestine [8], and as much as $90 \%$ of polyphenols arrive at the large intestine [9], where interactions may happen between polyphenols and gut microbiota. Polyphenols can be converted to bioactive compounds that may exert physiological effects by the gut microbiota, while the composition of microbiota can be modified by polyphenols [10]. Canarium album is a type of polyphenol-rich fruit with a total phenolic content of 1174.0-1799.6 mg gallic acid equivalents $/ 100 \mathrm{~g}$ fresh weight [11]. Its high polyphenol content is related to hepatoprotective [12], antioxidant [13], anti-inflammatory [14], and antiviral [15] effects. We previously found that polyphenol-rich Canarium album extract (CAext) restrains excessive lipid accumulation 
induced by oleic acid in hepatocarcinoma cells [16]. However, whether the pharmacological effects of polyphenols are attributed to its interactions with gut microbiota is not fully understood [10]. In light of this, the aim of the present study was to investigate the in vivo effects of CAext on the composition of intestinal microbiota of HFD-feeding mice.

\section{Results}

To understand the effect of CAext administration on the composition of gut microbiota, the gut microbiota composition was analyzed by Miseq sequencing on the V3-V4 region of 16S rRNA of bacteria. A total of 189,910 valid sequences and 146,325 operational taxonomic units (OTUs) were obtained after quality control, and the average length of each sequence was $442 \mathrm{bp}$.

Microbial phylotype richness was estimated by Chao and Ace, and the diversity of the bacterial community was revealed by Shannon [17]. As shown in Table 1, there was a significant overall decrease in the richness and the diversity of gut microbiota in the mice fed by HFD $(p<0.05)$. The NC group had the highest microbial phylotype richness and diversity in all groups, whereas the CAext groups had the lowest richness and diversity in all groups.

Table 1. Effect of CAext on the phylotype richness and the diversity of gut microbiota.

\begin{tabular}{cccccc}
\hline \multirow{2}{*}{ NC } & \multicolumn{4}{c}{ HFD } \\
\cline { 3 - 5 } & & MC & CAext-L & CAext-M & CAext-H \\
\hline Ace & $298.38 \pm 11.63^{\mathrm{a}}$ & $266.80 \pm 11.07^{\mathrm{b}, \mathrm{c}}$ & $272.74 \pm 14.05^{\mathrm{b}}$ & $251.69 \pm 13.70^{\mathrm{c}}$ & $222.70 \pm 10.44^{\mathrm{d}}$ \\
Chao1 & $304.65 \pm 19.48^{\mathrm{a}}$ & $269.87 \pm 15.92^{\mathrm{b}}$ & $279.94 \pm 22.79^{\mathrm{a}, \mathrm{b}}$ & $254.45 \pm 18.83^{\mathrm{b}}$ & $223.33 \pm 13.17^{\mathrm{c}}$ \\
Shannon & $4.19 \pm 0.02^{\mathrm{a}}$ & $3.73 \pm 0.02^{\mathrm{b}}$ & $3.07 \pm 0.02^{\mathrm{c}}$ & $3.20 \pm 0.02^{\mathrm{d}}$ & $3.54 \pm 0.02^{\mathrm{e}}$ \\
\hline
\end{tabular}

Data are presented as mean $\pm \mathrm{SD}$. NC, normal control, mice were fed with a normal chow and intragastrically administered $20 \mathrm{~mL} / \mathrm{kg}$ saline; MC, model control, mice were fed with a high-fat feed and intragastrically administered $20 \mathrm{~mL} / \mathrm{kg}$ saline; CAext-L, CAext-M, CAext-H mice were fed with a high-fat feed and intragastrically administered $10 \mathrm{mg} /(\mathrm{kg} \cdot \mathrm{d}), 15 \mathrm{mg} /(\mathrm{kg} \cdot \mathrm{d})$, and $20 \mathrm{mg} /(\mathrm{kg} \cdot \mathrm{d})$ Canarium album extract (CAext), respectively. Different letters indicate significant difference between treatment groups $(p<0.05)$.

Principal coordinate analysis (PCoA) based on the OTUs' abundance of different groups was performed to evaluate the similarities and differences among groups (Figure 1). The five groups were separated as two clusters along PC1 (57.67\%), suggesting that there were significant differences in the dominant bacterial population among the groups. The MC group was clearly separated along PC2 axis, indicating that CAext administration had a substantial effect on the gut microbial composition of HFD-fed mice. Cluster analysis of gut microbiota among different groups also had a similar result (Figure 2). 


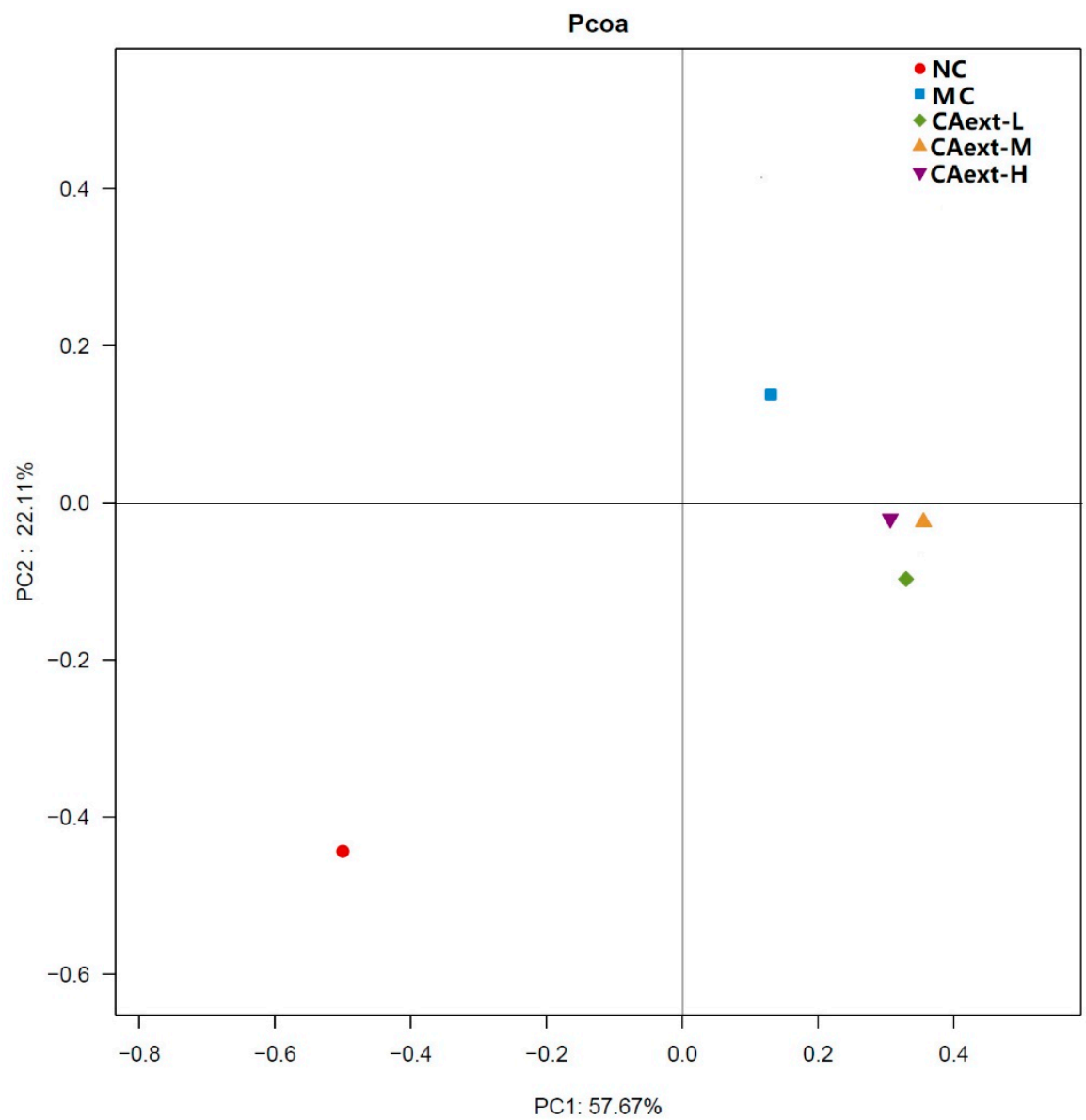

Figure 1. Principal coordinate analysis (PCoA) plots based on the OTU abundance of gut microbiota of each mouse. The closer the spatial distribution between spots, the greater the bacterial similarity among different groups. NC, normal control, mice were fed with a normal chow and intragastrically administered $20 \mathrm{~mL} / \mathrm{kg}$ saline; $\mathrm{MC}$, model control, mice were fed with a high-fat feed and intragastrically administered $20 \mathrm{~mL} / \mathrm{kg}$ saline; CAext-L, CAext-M, CAext-H mice were fed with a high-fat feed and intragastrically administered $10 \mathrm{mg} /(\mathrm{kg} \cdot \mathrm{d}), 15 \mathrm{mg} /(\mathrm{kg} \cdot \mathrm{d})$, and $20 \mathrm{mg} /(\mathrm{kg} \cdot \mathrm{d})$ Canarium album extract (CAext), respectively.

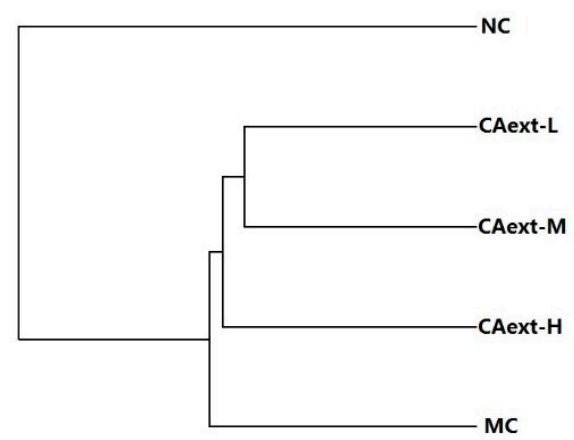

0.01

Figure 2. Clustering analysis dendrogram of gut microbiota based on distances among different groups. NC, normal control, mice were fed with a normal chow and intragastrically administered $20 \mathrm{~mL} / \mathrm{kg}$ saline; MC, model control, mice were fed with a high-fat feed and intragastrically administered $20 \mathrm{~mL} / \mathrm{kg}$ saline; CAext-L, CAext-M, CAext-H mice were fed with a high-fat feed and intragastrically administered $10 \mathrm{mg} /(\mathrm{kg} \cdot \mathrm{d}), 15 \mathrm{mg} /(\mathrm{kg} \cdot \mathrm{d})$, and $20 \mathrm{mg} /(\mathrm{kg} \cdot \mathrm{d})$ Canarium album extract (CAext), respectively. 
Analysis of relative bacterial abundance at the phylum level revealed the major differences of the dominant bacterial population among groups (Figure 3). Compared to the NC group, the relative abundance of Bacteroidetes was significantly reduced, whereas that of Firmicutes increased in all HFD-fed groups, especially in CAext-L and CAext-H group $(p<0.05)$. The Firmicutes to Bacteroidetes (F/B) ratio were respectively 0.50, 0.69, 1.79, 0.67, 1.09 in NC, MC, CAext-L, CAext-M, CAext-H group. Furthermore, the relative abundance of Verrucomicrobia was statistically higher in CAext-L and CAext-M groups compared with the NC or MC group $(p<0.05)$.

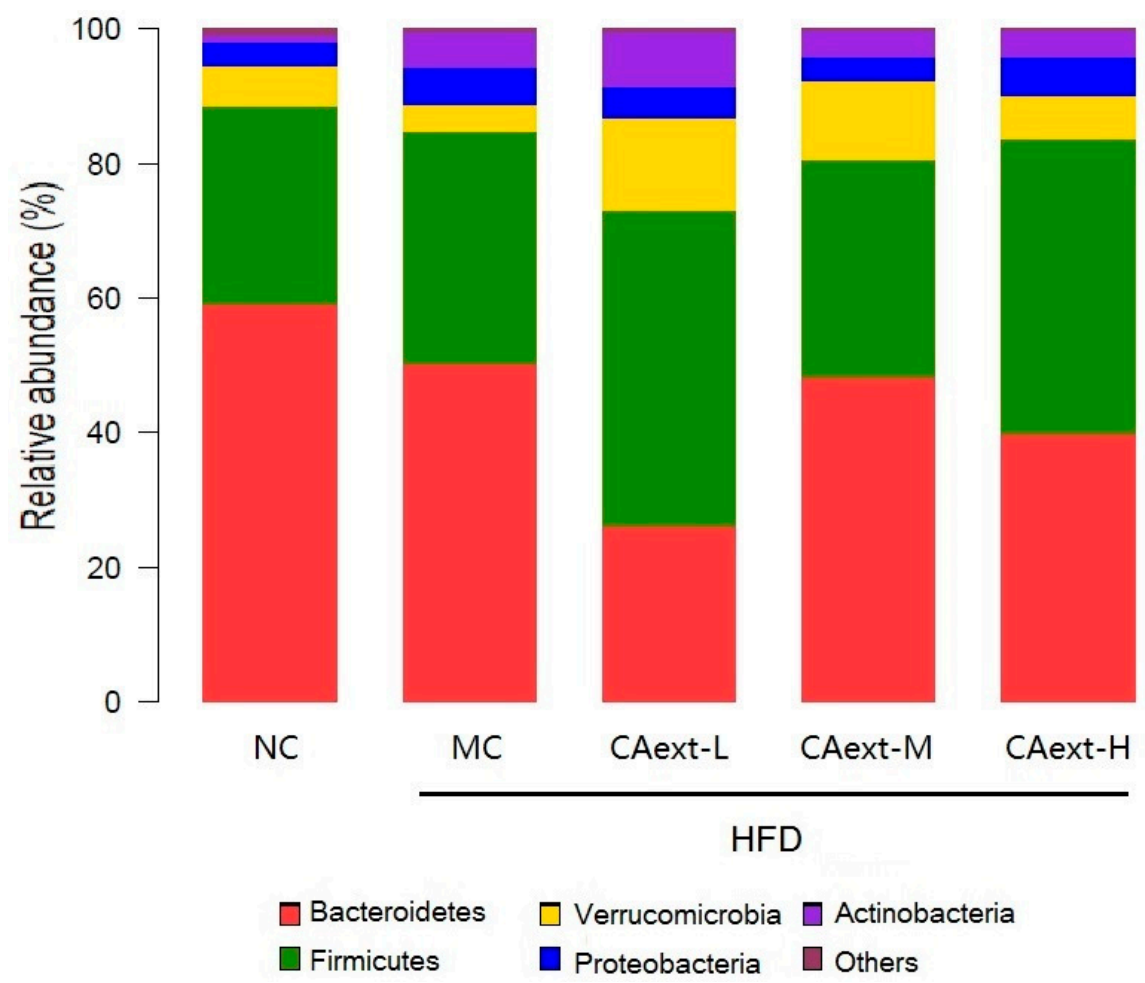

Figure 3. Relative abundance at the phylum level of gut microbiota among different groups. Other phyla refer to a taxa with a relative abundance $\leq 1 \%$ in any sample. NC, normal control, mice were fed with a normal chow and intragastrically administered $20 \mathrm{~mL} / \mathrm{kg}$ saline; MC, model control, mice were fed with a high-fat feed and intragastrically administered $20 \mathrm{~mL} / \mathrm{kg}$ saline; CAext-L, CAext-M, CAext-H mice were fed with a high-fat feed and intragastrically administered $10 \mathrm{mg} /(\mathrm{kg} \cdot \mathrm{d}), 15 \mathrm{mg} /(\mathrm{kg} \cdot \mathrm{d})$, and $20 \mathrm{mg} /(\mathrm{kg} \cdot \mathrm{d})$ Canarium album extract (CAext), respectively.

At the genus level (Figure 4), consumption of HFD statistically reduced the relative abundance of genus Bacteroidales_S24-7, which might be related to the reduction in the Bacteroidetes phylum, while the increase of the Firmicutes phylum might be attributed to the significant increase in the Allobaculum genus of all HFD-fed groups ( $p<0.05)$. Compared to the MC group, CAext administration significantly decreased the abundance of Bacteroidales_S24-7 $(p<0.05)$. However, no significant difference was obtained for the abundance of Allobaculum between MC and CAext groups $(p>0.05)$. As shown in Figure 4, CAext-L and CAext-M administration significantly increased the relative abundance of Akkermansia within the Verrucomicrobia phylum compared to the NC group or MC group (13.89 $\pm 3.78 \%$ in CAext-L, $11.68 \pm 4.12 \%$ in CAext-M vs. $6.01 \pm 3.92 \%$ in NC, $4.37 \pm 3.03 \%$ in $\mathrm{MC} ; p<0.05)$. 


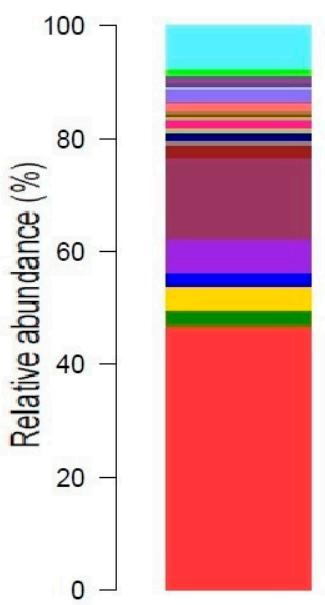

NC

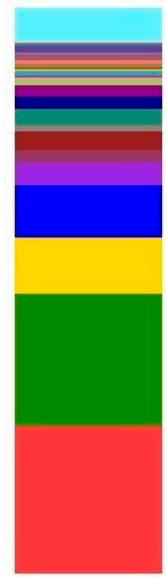

$\mathrm{MC}$

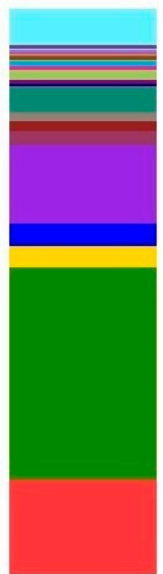

CAext-L

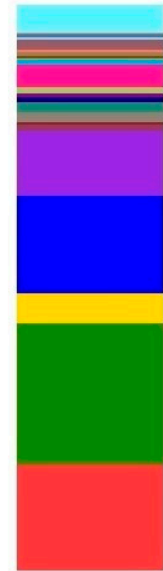

CAext-M

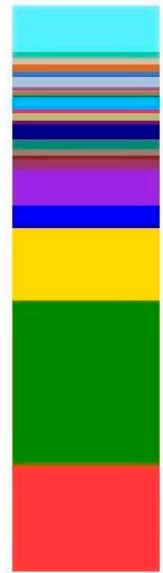

CAext-H

HFD

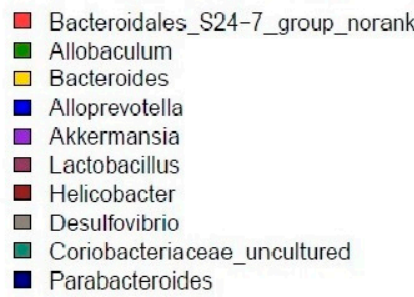

$\begin{array}{ll}\square \text { [Eubacterium]_coprostanoligenes_group } & \square \text { Lachnospiraceae_unclassified } \\ \square \text { Enterorhabdus } & \square \text { Romboutsia } \\ \square \text { Prevotellaceae_UCG-001 } & \square \text { Ruminococcaceae_UCG-014 } \\ \square \text { Parasutterella } & \square \text { Blautia } \\ \square \text { Anaerotruncus } & \square \text { Holdemania } \\ \square \text { Lachnospiraceae_uncultured } & \square \text { Ruminococcus_1 } \\ \square \text { Staphylococcus } & \square \text { Moryella } \\ \square \text { Alistipes } & \square \text { Anaerostipes } \\ \square \text { Prevotellaceae_unclassified } & \square \text { Others } \\ \square \text { Lachnospiraceae_NK4A136_group } & \end{array}$

Figure 4. Relative abundance at the genus level of gut microbiota among different groups. Other genera refer to a taxa with a relative abundance $\leq 1 \%$ in any sample. NC, normal control, mice were fed with a normal chow and intragastrically administered $20 \mathrm{~mL} / \mathrm{kg}$ saline; $\mathrm{MC}$, model control, mice were fed with a high-fat feed and intragastrically administered $20 \mathrm{~mL} / \mathrm{kg}$ saline; CAext-L, CAext-M, CAext-H mice were fed with a high-fat feed and intragastrically administered $10 \mathrm{mg} /(\mathrm{kg} \cdot \mathrm{d}), 15 \mathrm{mg} /(\mathrm{kg} \cdot \mathrm{d})$, and $20 \mathrm{mg} /(\mathrm{kg} \cdot \mathrm{d})$ Canarium album extract (CAext), respectively.

\section{Discussion}

HFD feeding can alter the diversity and composition of intestinal microbiota [18], and CAext administration further reshapes the gut microbiota of HFD-fed mice. Using high-throughput sequencing, we found significant changes in gut microbiota composition of different treatment groups. The richness and the diversity of gut microbiota significantly decreased in all HFD-fed groups (Table 1). Similarly, Xia et al. [19] investigated the effect of whole grain Qingke on intestinal microbiota of rats under HFD, and found that the alpha diversity indices were lower in the HFD group than in the other groups, and ascribed this decrease to dominant bacteria restraining others' growth. Bacteroidetes and Firmicutes are two major phyla dominant in most mammalian gut microbiota. Several studies have shown that obese subjects have a lesser proportion of Bacteroidetes, a higher proportion of Firmicutes, and a higher $\mathrm{F} / \mathrm{B}$ ratio compared to normal subjects $[8,20]$. Our results found that HFD loading induced a drop in Bacteroidetes and an increase in Firmicutes (Figure 3). Particularly, CAext administration was associated with a striking increase in the F/B ratio (0.50 in NC group vs. 1.79, 0.67, 1.09 in CAext-L, CAext-M, and CAext-H, respectively). Similar results were found by Anhe et al. [21], indicating that Firmicutes significantly increased and Bacteroidetes significantly decreased after polyphenol-rich cranberry extract treatment. Queipo-Ortuno et al. [22] found that both the concentration of Firmicutes and Bacteroidetes were significantly increased after a polyphenol-rich red wine period.

In the present study, we found that the relative abundance of Verrucomicrobia was statistically higher in CAext group compared with NC or MC group (Figure 3). This increasing of the proportion of Verrucomicrobia attributed to a significant increase of genus Akkermansia (Figure 4). Several studies have 
shown that different sources of dietary polyphenols increased the relative abundance of Akkermansia. Axling et al. [23] reported that green tea polyphenol increased the proportion of Akkermansia. Kemperman et al. [24] also found that complex polyphenols from black tea increase the relative abundance of Akkermansia. Akkermansia-like microorganisms are widely distributed in the intestines of the animals and human beings [25], and at least eight different species of the Akkermansia genus colonize the intestines of humans [26]. Akkermansia is a Gram-negative, strict anaerobe and mucin-degrading bacterium [27]. The beneficial effects of Akkermansia muciniphina have been extensively studied [28-30]. Multiple findings suggested that the improvement of metabolic disorders were associated with the increase of the Akkermansia population [31]. It has also been proved that Akkermansia administration has probiotic effects, which are associated with the ability of Akkermansia to preserve the mucus layer thickness [25], therefore reducing gut permeability [29], systemic lipopolysaccharide levels [21], host adiposity [32], and inflammatory markers [29].

Because of the tissue anatomical and physiological differences between species [33], animal models of HFD cannot fully replicate the complexity of human pathological conditions [34,35]. The results of this study may only provide a reference for studying the effect of CAext on the human microbial ecosystem. Another limitation is whether Akkermansia-related beneficial effects are sufficient to prevent the negative metabolic phenotype associated with major modifications in the proportions of Firmicutes and Bacteroidetes. Further research should be performed to clarify the effects of CAext on Akkermansia, and the mechanisms by which CAext or polyphenols reshape the gut microbiota with benefits to the host.

\section{Materials and Methods}

\subsection{Preparation of CAext}

CAext were prepared according to the method of Liu et al. [16]. Total polyphenol content was measured using the method of Giampieri et al. [36]. Total polyphenols content of CAext was $465.35 \pm 13.67 \mathrm{mg} / \mathrm{g}$.

\subsection{Animal Experiments}

All animal procedures were approved by the animal ethics committee of Fujian University of Traditional Chinese Medicine (Ethic Approve No. FJATCM-IAEC 2016012). Forty male KM mice (20 $\pm 2 \mathrm{~g})$, purchased from The Fujian Wus Laboratory Animal Co., Ltd. (Fujian, China), were housed in a room with a temperature of $24 \pm 2{ }^{\circ} \mathrm{C}$, a humidity of $55 \pm 5 \%$, and a 12:12 h light:dark cycle (8:00 am-8:00 pm).

The mice were fed a normal standard chow diet during their one week acclimatization period. After that, eight mice were randomly selected as the normal control group (NC), fed with a normal standard chow diet, and the rest of the thirty-two mice were fed with HFD for six weeks. According to Li et al. [37], the composition of the normal standard chow diet and the HFD with small changes are shown in Table 2. At the seventh week, the mice fed with HFD were randomly divided into the following four groups: model control group (MC, $n=8)$, low dose of CAext-treated group (CAext-L, $n=8)$, medium dose of CAext-treated group (CAext-M, $n=8)$, and high dose of CAext-treated group (CAext-H, $n=8$ ). The mice in MC and NC groups were treated with a dose of $20 \mathrm{~mL} / \mathrm{kg}$ saline once a day by gavage. The mice of the CAext-L, CAext-M, or CAext-H groups were orally administered a dose of $10 \mathrm{mg} / \mathrm{kg}, 15 \mathrm{mg} / \mathrm{kg}$, or $20 \mathrm{mg} / \mathrm{kg}$ CAext once a day, respectively. After four weeks of treatment with the above solution, all mice were sacrificed. The feces were collected and stored at $-80{ }^{\circ} \mathrm{C}$ for further analysis. 
Table 2. The composition of the normal standard chow diet and the HFD.

\begin{tabular}{ccc}
\hline Ingredients (g/100 g) & Normal Standard Chow Diet & HFD \\
\hline Corn starch & 30 & 26.3 \\
Wheat bran & 25 & 21.9 \\
Fish meal & 5 & 4.4 \\
Soy bean flour & 20 & 17.5 \\
Wheat flour & 16 & 14.0 \\
Yeast powder & 1 & 0.9 \\
Bone meal & 2 & 1.8 \\
Salt & 1 & 0.9 \\
Cholesterol & - & 2 \\
Lard oil & - & 10 \\
Sodium deoxycholate & - & 0.3 \\
\hline
\end{tabular}

\subsection{Fecal DNA Extraction}

Total DNA from fecal samples of eight mice per group was extracted using a TIANamp Stool DNA Kit (DP328, Tiangen Biotech (Bejing) Co., Ltd., Beijing, China). The concentration and the purity of DNA were determined using the NanoDrop 2000 spectrophotometer (Thermo Scientific, Waltham, MA, USA). The integrity of the extracted DNA was measured on $1.0 \%$ agarose gel.

\subsection{Fecal Microbiota Analysis}

The extracted DNA was used as a template to amplify the V3-V4 region of the bacteria $16 \mathrm{~S}$ rRNA gene (forward primer for V3 5'-ACTCCTACGGGAGGCAGCA-3', reverse primer for V4 $5^{\prime}$-GGACTACHVGGGTWTCTAAT-3'). The amplification program was as follows: initial denaturation at $95^{\circ} \mathrm{C}$ for $3 \mathrm{~min}$, amplification for 27 cycles of denaturation at $95^{\circ} \mathrm{C}$ for $30 \mathrm{~s}$, annealing at $55^{\circ} \mathrm{C}$ for $30 \mathrm{~s}$, and extension at $72{ }^{\circ} \mathrm{C}$ for $45 \mathrm{~s}$, then extension at $72{ }^{\circ} \mathrm{C}$ for $10 \mathrm{~min}$.

Sequencing was performed according to the protocol of Illumina Miseq platforms at Shanghai Majorbio Bio-Pharm Technoloy Co., Ltd., Shanghai, China.

To obtain high-quality reads, the raw reads were quality filtered using QIIME software (Version 1.9.0, http://qiime.org). The chimeric sequences were identified and removed with the UCHIME algorithm (UCHIME Algorithm, http:/ / www.drive5.com/usearch/manual/uchime_algo. $\mathrm{html})$. The remaining sequences with $97 \%$ identity were clustered into the same operational taxonomic units (OTUs) using Usearch software (Version 7.1, http:/ / drive5.com/usearch/). The representative sequences of each OUT were assigned to RDP classifier (Version 2.2, http://rdp.cme.msu.edu/ classifier). Alpha diversity index based on the OTUs was conducted by Mothur software (Version 1.30.0, http:/ / www.mothur.org). All the statistical analyses of the sequenced data were performed by using the R software package (Version 3.2.3, http:/ / www.R-project.org).

\subsection{Statistical Analysis}

Results are represented as mean \pm SD. Statistical analysis was performed through one-way analysis of variance (ANOVA) using IBM SPSS 22.0 (IBM, Armonk, NY, USA), $p<0.05$ was considered statistically significant.

\section{Conclusions}

In conclusion, administration of polyphenol-rich CAext altered the composition of the gut microbiota with an increase in the relative abundance of Firmicutes and Verrucomicrobia, along with a decrease in Bacteroidetes. Furthermore, CAext-L and CAext-M greatly increased the population of the Akkermansia. Further studies will elucidate the prebiotic effect of CAext on the gut microbiota. 
Author Contributions: Conceptualization, N.-N.Z., B.-D.Z. and S.-X.Z.; Methodology, N.-N.Z. and W.-H.G.; Software, H.H.; Formal Analysis, N.-N.Z., W.-H.G. and A.-R.Z.; Investigation, N.-N.Z. and Q.-P.L.; Data Curation, N.-N.Z.; Writing-Original Draft Preparation, N.-N.Z.; Writing-Review \& Editing, S.-X.Z.; Supervision, B.-D.Z.; Funding Acquisition, S.-X.Z.

Funding: This research was funded by Science and Technology Program of the Fujian Province, China (Grant No. 2015N3009).

Conflicts of Interest: The authors declare no conflict of interest.

\section{References}

1. Zhao, Y.; Wu, J.F.; Li, J.V.; Zhou, N.Y.; Tang, H.R.; Wang, Y.L. Gut microbiota composition modifies fecal metabolic profiles in mice. J. Proteome Res. 2013, 12, 2987-2999. [CrossRef] [PubMed]

2. Zhang, C.; Zhang, M.; Wang, S.; Han, R.; Cao, Y.; Hua, W.; Mao, Y.; Zhang, X.; Pang, X.; Wei, C.; et al. Interactions between gut microbiota, host genetics and diet relevant to development of metabolic syndromes in mice. ISME J. 2010, 4, 232-241. [CrossRef] [PubMed]

3. Cuervo, A.; Valdés, L.; Salazar, N.; de los Reyes-Gavilán, C.G.; Ruas-Madiedo, P.; Gueimonde, M.; González, S. Pilot study of diet and microbiota: Interactive associations of fibers and polyphenols with human intestinal bacteria. J. Agric. Food Chem. 2014, 62, 5330-5336. [CrossRef] [PubMed]

4. Cuervo, A.; Arboleya, S.; Gueimonde, M.; González, S. Microbiota modulation by diet in humans. Prebiotics, fibres and other compouds. Agro Food Ind. Hi-Tech. 2012, 23, 23-26.

5. Hildebrandt, M.A.; Hoffmann, C.; Sherrill-Mix, S.A.; Keilbaugh, S.A.; Hamady, M.; Chen, Y.Y.; Knight, R.; Ahima, R.S.; Bushman, F.; Wu, G.D. High-fat diet determines the composition of the murine gut microbiome independently of obesity. Gastroenterology 2009, 137, 1716-1724. [CrossRef] [PubMed]

6. Serino, M.; Luche, E.; Gres, S.; Baylac, A.; Bergé, M.; Cenac, C.; Waget, A.; Klopp, P.; Iacovoni, J.; Klopp, C.; et al. Metaboic adaptation to a high-fat diet is associated with a change in the gut microbiota. Gut 2012, 61, 543-553. [CrossRef] [PubMed]

7. Everard, A.; Lazarevic, V.; Derrien, M.; Girard, M.; Muccioli, G.G.; Neyrinck, A.M.; Possemiers, S.; Van Holle, A.; Francois, P.; de Vos, W.M.; et al. Responses of gut microbiota and glucose and lipid metabolism to prebiotics in genetic obese and diet-induced leptin-resistant mice. Diabetes 2011, 60, 2775-2786. [CrossRef] [PubMed]

8. Magistrelli, D.; Zanchi, R.; Malagutti, L.; Galassi, G.; Canzi, E.; Rosi, F. Effects of cocoa husk feeding on the composition of swine intestinal microbiota. J. Agric. Food Chem. 2016, 64, 2046-2052. [CrossRef] [PubMed]

9. Etxeberria, U.; Fernandez-Quintela, A.; Milagro, F.I.; Aguirre, L.; Martinez, J.A.; Portillo, M.P. Impact of polyphenols and polyphenol-rich dietary sources on gut microbiota composition. J. Agric. Food Chem. 2013, 61, 9517-9533. [CrossRef] [PubMed]

10. Larrosa, M.; Luceri, C.; Vivoli, E.; Pagliuca, C.; Lodovici, M.; Moneti, G.; Dolara, P. Polyphenol metabolites from colonic microbiota exert anti-inflammatory activity on different inflammation models. Mol. Nutr. Food Res. 2009, 53, 1044-1054. [CrossRef] [PubMed]

11. Chang, Q.; Su, M.H.; Chen, Q.X.; Zeng, B.Y.; Li, H.H.; Wang, W. Physicochemical properties and antioxidant capacity of Chinese olive (Canarium album L.) cultivars. J. Food Sci. 2017, 82, 1369-1377. [CrossRef] [PubMed]

12. Ito, M.; Shimura, H.; Watanabe, N.; Tamai, M.; Hanada, K.; Takahashi, A.; Tanaka, Y.; Arai, K.; Zhang, P.L.; Chang, R. Hepatoprotective compounds from Canarium album and Euphorbia nematocypha. Chem. Pharm. Bull. 1990, 38, 2201-2203. [PubMed]

13. Liu, H.; Qiu, N.; Ding, H.; Yao, R. Polyphenols contents and antioxidant capacity of 68 Chinese herbals suitable for medical or food uses. Food Res. Int. 2008, 41, 363-370. [CrossRef]

14. Santino, A.; Scarano, A.; Santis, S.; Benedictis, M.; Giovinazzo, G.; Chieppa, M. Gut microbiota modulation and anti-inflammatory properties of dietary polyphenols in IBD: New and consolidated perspectives. Curr. Pharm. Des. 2017, 23, 2344-2351. [CrossRef] [PubMed]

15. Duan, W.; Tan, S.; Chen, J.; Liu, S.; Jiang, S.; Xiang, H.; Xie, Y. Isolation of anti-HIV components from Canarium album fruits by high-speed counter-current chromatography. Anal. Lett. 2013, 46, 1057-1068. [CrossRef]

16. Liu, Q.; Zhou, M.; Zheng, M.; Chen, N.; Zheng, X.; Zeng, S.; Zheng, B. Canarium album extract restrains lipid excessive accumulation in hepatocarcinoma cells. Int. J. Clin. Exp. Med. 2016, 9, 17509-17518. 
17. Dong, W.W.; Xuan, F.L.; Zhong, F.L.; Jiang, J.; Wu, S.; Li, D.; Li, D. Comparative analysis of the rats' gut microbiota composition in animals with different ginsenosides metabolizing activity. J. Agric. Food Chem. 2017, 65, 327-337. [CrossRef] [PubMed]

18. Qiao, Y.; Sun, J.; Ding, Y.; Le, G.; Shi, Y. Alterations of the gut microbiota in high-fat diet mice is strongly linked to oxidative stress. Appl. Microbiol. Biotechnol. 2013, 97, 1689-1698. [CrossRef] [PubMed]

19. Xia, X.; Li, G.; Ding, Y.; Ren, T.; Zheng, J.; Kan, J. Effect of whole grain Qingke (Tibetan Hordeum vulgare L. Zangqing 320) on the serum lipid levels and intestinal microbiota of rats under high-fat diet. J. Agric. Food Chem. 2017, 65, 2686-2693. [CrossRef] [PubMed]

20. Ley, R.E.; Turnbaugh, P.J.; Klein, S.; Gordon, J.I. Microbial ecology: Human gut microbes associated with obesity. Nature 2006, 444, 1022-1023. [CrossRef] [PubMed]

21. Anhe, F.F.; Roy, D.; Pilon, G.; Dudonne, S.; Matamoros, S.; Varin, T.V.; Garofalo, C.; Moine, Q.; Desjardins, Y.; Levy, E.; et al. A polyphenol-rich cranberry extract protects from diet-induced obesity, insulin resistance and intestinal inflammation in association with increased Akkermansia spp. population in the gut microbiota of mice. Gut 2015, 64, 872-883. [CrossRef] [PubMed]

22. Queipo-Ortuno, M.I.; Boto-Ordonez, M.; Murri, M.; Gomez-Zumaquero, J.M.; Clemente-Postigo, M.; Estruch, R.; Cardona Diaz, F.; Andres-Lacueva, C.; Tinahones, F.J. Influence of red wine polyphenols and ethanol on the gut microbiota ecology and biochemical biomarkers. Am. J. Clin. Nutr. 2012, 95, 1323-1334. [CrossRef] [PubMed]

23. Axling, U.; Olsson, C.; Xu, J.; Fernandez, C.; Larsson, S.; Ström, K.; Ahrné, S.; Holm, C.; Molin, G.; Berger, K. Green tea powder and Lactobacillus plantarum affect gut microbiota, lipid metabolism and inflammation in high-fat fed C57BL/6J mice. Nutr. Metab. 2012, 9, 105. [CrossRef] [PubMed]

24. Kemperman, R.A.; Gross, G.; Mondot, S.; Possemiers, S.; Marzorati, M.; Van de Wiele, T.; Doré, J.; Vaughan, E.E. Impact of polyphenols from black tea and red wine/grape juice on a gut model microbiome. Food Res. Int. 2013, 53, 659-669. [CrossRef]

25. Belzer, C.; de Vos, W.M. Microbes inside-from diversity to function: The case of Akkermansia. ISME J. 2012, 6, 1449-1458. [CrossRef] [PubMed]

26. Van den Abbeele, P.; Gérard, P.; Rabot, S.; Bruneau, A.; El Aidy, S.; Derrien, M.; Kleerebezem, M.; Zoetendal, E.G.; Simdt, H.; Verstraete, W.; et al. Arabinoxylans and inulin differentially modulate the mucosal and luminal gut microbiota and mucin-degradation in humanized rats. Environ. Microbiol. 2011, 13, 2667-2680. [CrossRef] [PubMed]

27. Derrien, M.; Collado, M.C.; Ben-Amor, K.; Salminen, S.; de Vos, W.M. The mucin degrader Akkermansia muciniphila is an abundant resident of the human intestinal tract. Appl. Environ. Microbiol. 2008, 74, 1646-1648. [CrossRef] [PubMed]

28. Mei, X.; Zhang, X.; Wang, Z.; Gao, Z.; Liu, G.; Hu, H.; Zou, L.; Li, X. Insulin sensitivity-enhancing activity of phlorizin is associated with lipopolysaccharide decrease and gut microbiota changes in obese and type 2 diabetes (db/db) Mice. J. Agric. Food Chem. 2016, 64, 7502-7511. [CrossRef] [PubMed]

29. Everard, A.; Belzer, C.; Geurts, L.; Ouwerkerk, J.P.; Druart, C.; Bindels, L.B.; Guiot, Y.; Derrien, M.; Muccioli, G.G.; Delzenne, N.M.; et al. Cross-talk between Akkermansia muciniphila and intestinal epithelium controls diet-induced obesity. Proc. Natl. Acad. Sci. USA 2013, 110, 9066-9071. [CrossRef] [PubMed]

30. Shin, N.R.; Lee, J.C.; Lee, H.Y.; Kim, M.S.; Whon, T.W.; Lee, M.S.; Bae, J.W. An increase in the Akkermansia spp. population induced by metformin treatment improves glucose homeostasis in diet-induced obese mice. Gut 2014, 63, 727-735. [CrossRef] [PubMed]

31. Shen, W.; Shen, M.; Zhao, X.; Zhu, H.; Yang, Y.; Lu, S.; Tan, Y.; Li, G.; Li, M.; Wang, J.; et al. Anti-obesity effect of capsaicin in mice fed with high-fat diet is associated with an increase in population of the gut bacterium Akkermansia muciniphila. Front. Microbiol. 2017, 8, 272. [CrossRef] [PubMed]

32. Roopchand, D.E.; Carmody, R.N.; Kuhn, P.; Moskal, K.; Rojas-Silva, P.; Turnbaugh, P.J.; Raskin, I. Dietary polyphenols promote growth of the gut bacterium Akkermansia muciniphila and attenuate high-fat diet-induced metabolic syndrome. Diabetes 2015, 64, 2847-2858. [CrossRef] [PubMed]

33. Yu, B.; West, L.; Moussy, Y.; Moussy, F. Transcutaneous implantation methods for improving the long-term performance of glucose sensors in rats. IEEE Sens. J. 2008, 8, 97-103. [CrossRef]

34. Caddeo, S.; Boffito, M.; Sartori, S. Tissue engineering approaches in the design of healthy and pathological in vitro tissue models. Front. Bioeng. Biotechnol. 2017, 5, 40. [CrossRef] [PubMed] 
35. Suzuki, Y.; Yeung, A.C.; Ikeno, F. The representative porcine model for human cardiovascular disease. J. Biomed. Biotechnol. 2015, 2011, 195483. [CrossRef] [PubMed]

36. Giampieri, F.; Alvarez-Suarez, J.M.; Mazzoni, L.; Forbes-Hernandez, T.Y.; Gasparrini, M.; Gonzàlez-Paramàs, A.M.; Santos-Buelga, C.; Quiles, J.L.; Bompadre, S.; Mezzetti, B.; et al. Polyphenol-rich strawberry extract protects human dermal fibroblasts against hydrogen peroxide oxidative damage and improves mitochondrial functionality. Molecules 2014, 19, 7798-7816. [CrossRef] [PubMed]

37. Li, F.; Li, D.Y. Study on the effect of olive oil on hyperlipidemia in model rats. China Trop. Med. 2007, 7, 2136-2137.

Sample Availability: Samples of the compounds are not available from the authors.

(C) 2018 by the authors. Licensee MDPI, Basel, Switzerland. This article is an open access article distributed under the terms and conditions of the Creative Commons Attribution (CC BY) license (http://creativecommons.org/licenses/by/4.0/). 